\title{
ENDOMORPHISM MONOIDS OF DISTRIBUTIVE DOUBLE P-ALGEBRAS
}

\author{
by M. E. ADAMS $\dagger$ and J. SICHLER $\dagger$
}

(Received 17 September, 1977)

1. Introduction. A distributive p-algebra is an algebra $\langle L ; \vee, \wedge, *, 0,1\rangle$ for which $\langle L, \vee, \wedge, 0,1\rangle$ is a bounded distributive lattice and ${ }^{*}$ is a unary operation on $L$ such that $a \wedge x=0$ if and only if $x \leq a^{*}$ (i.e. a pseudocomplementation). A distributive double $p$-algebra is an algebra $\left\langle L ; \vee, \wedge,{ }^{*},{ }^{+}, 0,1\right\rangle$ in which the deletion of ${ }^{+}$gives a distributive p-algebra and the deletion of $*$ gives a dual distributive p-algebra, that is $a \vee x=1$ if and כnly if $x \geq a^{+}$.

In the following it will be shown that for any finite monoid $M$ there exists a finite distributive double p-algebra $L$ whose endomorphism monoid $\operatorname{End}(L)$ is isomorphic to $M$.

The general question of whether for any given monoid $M$ there exists a distributive double p-algebra $L$ such that $\operatorname{End}(L) \cong M$ remains open. However, it will be shown that there exist rigid distributive double p-algebras of arbitrarily large cardinality. (An algebra $L$ is rigid if and only if $|\operatorname{End}(L)|=1$.)

2. Preliminaries. A directed graph is a pair $(X, R)$ in which $R$ is a set of ordered pairs of distinct elements of $X$. A mapping $f: X \rightarrow X^{\prime}$ for which $\left\langle f\left(x_{1}\right), f\left(x_{2}\right)\right\rangle \in R^{\prime}$ for every $\left\langle x_{1}, x_{2}\right\rangle \in R$ is called a compatible mapping of the directed graph $(X, R)$ into the directed graph $\left(X^{\prime}, R^{\prime}\right)$.

An ordered space $(S, \tau, \leq)$ is a set $S$, with a topology $\tau$, and a partial order relation $\leq$. A subset $X$ of a partially ordered set $S$ is decreasing if and only if, for all $x \in X, y \leq x$ implies that $y \in X$; increasing is defined dually. An ordered space $(S, \tau, \leq)$ is totally order disconnected if and only if, for $x, y \in S, x \neq y$ implies that there exists a clopen decreasing set $X$ such that $x \in X$ and $y \notin X$. If $S_{1}, S_{2}$ are partially ordered sets then a mapping $f: S_{1} \rightarrow S_{2}$ is order preserving if and only if for $x, y \in S_{1}, x \leq y$ implies that $f(x) \leq f(y)$.

H. A. Priestley has used ordered spaces to give a duality theory for the category of bounded distributive lattices.

Theorem 1 (H. A. Priestley [8]). The category of $(0,1)$-distributive lattices with $(0,1)$-lattice homomorphisms is dual (that is, isomorphic under a contravariant functor) to the category of compact totally order disconnected spaces with continuous order preserving maps.

Each $(0,1)$-distributive lattice $L$ is isomorphic to the lattice of clopen decreasing subsets of its dual space $\mathscr{S}(L)$.

Let $S$ be a poset; for $x \in S$ let $\operatorname{Min}(x)=\{y \mid y \leq x$ and $y$ is minimal $\}$ and $\operatorname{Max}(x)=$ $\{y \mid y \geq x$ and $y$ is maximal $\}$. For $X \subseteq S$, let $\operatorname{Max}(X)=\cup\{\operatorname{Max}(x) \mid x \in X\} ; \operatorname{Min}(X)$ is defined dually. The Priestley duality can be used to recognize those $(0,1)$-distributive

† The support of the National Research Council of Canada is gratefully acknowledged.

Glasgow Math. J. 20 (1979) 81-86. 
lattices possessing pseudocomplements and dual pseudocomplements and to characterize their homomorphisms as distributive double p-algebras; the duality for distributive double p-algebras follows from H. A. Priestley [10].

Proposition 2. Let $\mathscr{S}(L)$ be a compact totally order disconnected space.

(i) $L$ is a distributive double $p$-algebra if and only if for every clopen decreasing set $X$, $[X)$ is open in $\mathscr{S}(L)$, and for every clopen increasing set $Y,(Y]$ is open in $\mathscr{S}(L)$.

(ii) Let $\varphi: L_{1} \rightarrow L_{2}$ be a $(0,1)$-lattice homomorphism between the distributive double $p$-algebras $L_{1}$ and $L_{2}$. Then $\varphi$ is a double p-homomorphism if and only if the corresponding continuous order preserving map $f: \mathscr{S}\left(L_{2}\right) \rightarrow \mathscr{S}\left(L_{1}\right)$ satisfies $f(\operatorname{Min}(x))=\operatorname{Min}(f(x))$ and $f(\operatorname{Max}(x))=\operatorname{Max}(f(x))$ for all $x \in \mathscr{S}\left(L_{2}\right)$.

Observe that if $L$ is a distributive double p-algebra, then each clopen decreasing set in $\mathscr{S}(L)$ corresponds to an element of the algebra. Let $X$ be a clopen decreasing subset of $\mathscr{S}(L)$, then $X^{*}=\mathscr{S}(L) \backslash[X)$ and $X^{+}=(\mathscr{S}(L) \backslash X]$.

Further information on distributive double $\mathrm{p}$-algebras may be found in $\mathrm{R}$. Beazer [1] and T. Katriňak [5]; the duality is considered in B. A. Davey [2].

We would like to acknowledge stimulating conversations with $R$. Beazer who also suggested an investigation of distributive double p-algebras to us.

3. Representation of finite monoids. Let $(X, R)$ be a finite directed graph for which both the domain and the range of $R$ are equal to $X$. We will define a finite partially ordered set $P(X, R)$ associated with $(X, R)$.

Let $X_{0}, X_{1}$ be two distinct copies of $X$; for $x \in X$, the corresponding element of $X_{i}$ will be denoted by $x_{(i)}$. For $i=0, \ldots, 8$, choose new elements $a_{i}$. For $i=0, \ldots, 7$, choose new elements $b_{i}$. Set $A=\left\{a_{0}, \ldots, a_{8}\right\}$ and $B=\left\{b_{0}, \ldots, b_{7}\right\}$. The domain of $P(X, R)$ is the set

\section{$A \cup B \cup X \cup R \cup X_{0} \cup X_{1}$.}

A partial ordering relation is defined over this set as the transitive closure of the following relation:

$$
\begin{aligned}
& a_{0}<b_{0}, b_{0}>a_{1}, \ldots, a_{n}<b_{n}, b_{n}>a_{n+1}, \ldots, b_{7}>a_{8} \text {, } \\
& x_{(1)}<b_{0} \text { and } b_{1} \quad \text { for } x \in X \text {, } \\
& \langle x, y\rangle\left\langle b_{2} \text { and } b_{3} \text { for }\langle x, y\rangle \in R\right. \text {, } \\
& a_{5} \text { and } a_{6}<x \quad \text { for } \quad x \in X \text {, } \\
& a_{7} \text { and } a_{8}<x_{(0)} \quad \text { for } \quad x \in X \text {, } \\
& x \text { and } x_{(0)}<x_{(1)} \quad \text { for } \quad x \in X \text {, } \\
& x \text { and } y_{(0)}<\langle x, y\rangle \text { for }\langle x, y\rangle \in R \text {. }
\end{aligned}
$$

It is a matter of checking to see that the transitive closure of the above relation is indeed a partial ordering; intuitively, the four sets $X, X_{0}, X_{1}$, and $R$ are added to a fence with a prescribed order relation between them. 
Endowed with the trivial topology the finite partially ordered set $P(X, R)$ is the dual space of a finite distributive lattice, and hence of a finite distributive double p-algebra. Let $\operatorname{DP}(X, R)$ denote the distributive double p-algebra whose dual space is $P(X, R)$. Using Proposition 2 we now investigate the endomorphisms of $\operatorname{DP}(X, R)$.

LEMMA 1. Let $f: P(X, R) \rightarrow P(X, R)$ be the dual of an endomorphism of $\operatorname{DP}(X, R)$. Then $f(a)=a$ for $a \in A$ and $f(b)=b$ for $b \in B$.

Proof. By definition, $\operatorname{Min}(P(X, R))=A$ and $\operatorname{Max}(P(X, R))=B$. Thus, by Proposition 2, $f(A) \subseteq A$ and $f(B) \subseteq B$. The poset $P(X, R)$ is connected (i.e. for $x, y \in P(X, R)$, there exist $x_{i}, i=0, \ldots, n$, such that $x=x_{0}, x_{0} \leq x_{1}, x_{1} \geq x_{2}, \ldots, x_{2 j} \leq x_{2 j+1}, x_{2 j+1} \geq$ $\left.x_{2(j+1)}, \ldots, x_{n}=y\right)$. The connectivity together with Proposition 2 can be used to show that $A \subseteq f(A)$ and $B \subseteq f(B)$. Since $P(X, R)$ is finite the restrictions of $f$ to the sets $A$ and $B$ are bijections. Thus, $|\operatorname{Max}(a)|=|\operatorname{Max}(f(a))|$ for $a \in A$ and $|\operatorname{Min}(b)|=|\operatorname{Min}(f(b))|$ for $b \in B$. In particular, since $\left|\operatorname{Max}\left(a_{0}\right)\right|=1$ and $a_{0}$ is the only element of $A$ with this property, we deduce that $f\left(a_{0}\right)=a_{0}$. The mapping $f$ is order preserving. Thus, $f\left(b_{0}\right) \in \operatorname{Max}\left(a_{0}\right)$; that is $f\left(b_{0}\right)=b_{0}$. In consequence, $f\left(a_{1}\right) \in \operatorname{Min}\left(b_{0}\right)=\left\{a_{1}, a_{5}, a_{6}, a_{7}, a_{8}\right\}$. However, $\left|\operatorname{Max}\left(a_{1}\right)\right|=2$ and $a_{1}$ is singled out from $\operatorname{Min}\left(b_{0}\right)$ by this property. Whence, $f\left(a_{1}\right)=a_{1}$. By similar reasoning, we inductively show that first $f\left(b_{i}\right)=b_{i}$ and then $f\left(a_{i+1}\right)=a_{i+1}$ for $i=1, \ldots, 7$.

LEMMA 2. Let $f: P(X, R) \rightarrow P(X, R)$ be the dual of an endomorphism of $\operatorname{DP}(X, R)$. Then $x \in R$ implies $f(x) \in R, x \in X$ implies $f(x) \in X$, and, for $i=0,1, x \in X_{i}$ implies $f(x) \in X_{i}$.

Proof. For $x \in P(X, R), x \in R$ if and only if $x \leq b_{2}, x \leq b_{3}, a_{5} \leq x, a_{6} \leq x, a_{7} \leq x$, and $a_{8} \leq x$. The mapping $f$ is order preserving. Thus, by Lemma $1, f(x) \in R$.

Similarly, if $x \in X$ then $f(x) \in X$ and, for $i=0,1$ if $x \in X_{i}$ then $f(x) \in X_{i}$.

LEMMA 3. Let $f: P(X, R) \rightarrow P(X, R)$ be the dual of an endomorphism of $\operatorname{DP}(X, R)$. Then the restriction of $f$ to $X$ defines a compatible mapping of the directed graph $(X, R)$ into itself.

Proof. By Lemma 2, $x \in X$ implies that $f(x) \in X$. Thus $f$ is well defined on the vertex set of the directed graph $(X, R)$.

It remains to be shown that if $\langle x, y\rangle \in R$ then $\langle f(x), f(y)\rangle \in R$. First we show that if $x \in X$ then $f\left(x_{(0)}\right)=(f(x))_{(0)}$. By definition, $x<x_{(1)}$. Thus, $f(x)<f\left(x_{(1)}\right) \in X_{1}$; that is $f\left(x_{(1)}\right)=$ $(f(x))_{(1)}$. Similarly, from $x_{(1)}>x_{(0)}$ we deduce that $f\left(x_{(1)}\right)=(f(x))_{(1)}>f\left(x_{(0)}\right) \in X_{0}$. Hence, $f\left(x_{(0)}\right)=(f(x))_{(0)}$. If $\langle x, y\rangle \in R$ then $x, y_{(0)}<\langle x, y\rangle$ in $P(X, R)$. By Lemma $2, f(x) \in X, f\left(y_{(0)}\right)=$ $(f(y))_{(0)} \in X_{0}$, and $f(\langle x, y\rangle)=\left\langle x^{\prime}, y^{\prime}\right\rangle \in R$ for some ordered pair $\left\langle x^{\prime}, y^{\prime}\right\rangle$. However, $f(x),(f(y))_{(0)}<\left\langle x^{\prime}, y^{\prime}\right\rangle$ if and only if $x^{\prime}=f(x)$ and $y^{\prime}=f(y)$. We conclude that $\langle f(x), f(y)\rangle \in$ $\boldsymbol{R}$.

Lemma 4. Let $f:(X, R) \rightarrow(X, R)$ be a compatible mapping of the directed graph $(X, R)$. Then $f$ has a unique extension to a mapping of $P(X, R)$ into itself which is the dual of an endomorphism of $\operatorname{DP}(X, R)$.

Proof. Using Lemma 1 and Lemma 2, we extend $f$ to a mapping of $P(X, R)$ into itself in the only way possible: $f(a)=a$ for $a \in A$ and $f(b)=b$ for $b \in B$. For $i=0,1$, set 
$f\left(x_{(i)}\right)=(f(x))_{(i)}$. Finally, for $\langle x, y\rangle \in R$, let $f(\langle x, y\rangle)=\langle f(x), f(y)\rangle$; since $f$ is a compatible mapping this is always possible. The extended mapping $f$ is continuous (since $P(X, R)$ is finite), order preserving, and satisfies condition (ii) of Proposition 2.

Lemma 3 and Lemma 4 combine to the following:

Lemma 5. Let $(X, R)$ be a finite directed graph and $\operatorname{DP}(X, ' R)$ the associated finite distributive double p-algebra. Then $\operatorname{End}(\operatorname{DP}(X, R))$ is dually isomorphic to $\operatorname{End}(X, R)$.

It follows from [4] or [12] that for any finite monoid $M$ there exist infinitely many non-isomorphic finite directed graphs (whose vertices are both elements of the domain and the range of the set of edges) for each of which the endomorphism monoid of compatible mappings is isomorphic to $M$. We conclude:

THEOREM 3. Let $M$ be a finite monoid. There exist infinitely many non-isomorphic finite distributive double $p$-algebras $L_{i}, i=0,1,2, \ldots$ such that $\operatorname{End}\left(L_{i}\right) \cong M, i=0,1,2, \ldots$.

4. Rigid distributive double p-algebras. Let $L$ be a chain with a greatest and a least element. Then $C=\mathscr{T}(L)$ is a compact totally order disconnected space; $C$ is totally ordered by the partial order on the space. For $i=0,1$, choose topological spaces $C_{i}$ homeomorphic to $C$. If $f: C \rightarrow C_{i}$ is a homeomorphism, let $c_{(i)}$ denote $f(c)$ for $c \in C$; for $D \subseteq C$, let $D_{(i)}=\left\{d_{(i)} \mid d \in D\right\}$. Since $C, C_{0}$, and $C_{1}$ are compact totally disconnected spaces the disjoint union $S(C)$ is also a compact totally disconnected space. We define a partial order on $S(C)$ in the following manner:

$$
\text { for } c \leq d \text { in } C, \quad c_{(0)}<c \leq d<d_{(1)} \text {. }
$$

Observe that $C_{0}=\operatorname{Min}(S(C))$ and $C_{1}=\operatorname{Max}(S(C))$.

Lemma 6. The space $S(C)$ is the dual of a distributive double p-algebra.

Proof. Let $x, y \in S(C)$ with $x \neq y$. The subspaces $C, C_{0}$ and $C_{1}$ are each totally order disconnected. Further, $C_{0}$ and $C_{1}$ are totally unordered. Thus, if either $x \in C_{0}$ or $y \in C_{1}$ we may choose a clopen decreasing set $X \subseteq S(C)$ such that $x \in X$ and $y \in X$. Let us suppose that $x \notin C_{0}$ and $y \notin C_{1}$. If $x \in S(C)$, then $x \in\left\{c, c_{(0)}, c_{(1)}\right\}$ for some $c \in C$; set $x^{\prime}=c$. By assumption $x^{\prime} \geq y^{\prime}$. Thus there exists a clopen decreasing set $X^{\prime} \subseteq C$ such that $x^{\prime} \in X^{\prime}$ and $y^{\prime} \notin X^{\prime}$. It follows that $x \in X$ and $y \notin X$, for the clopen decreasing set $X=X^{\prime} \cup X_{(0)}^{\prime} \cup X_{(1)}^{\prime}$. We conclude that $S(C)$ is totally order disconnected.

It remains to be shown that the compact totally order disconnected space $S(C)$ satisfies the condition of Proposition 2(i). Let $X$ be a clopen decreasing set. If $X \cap C \neq \varnothing$, then $[X)=X \cup C \cup C_{1}$ is an open set. Alternatively, if $X \cap C=\varnothing$ then $X=D_{(0)}$. for some open set $D \subseteq C$. However, the chain $L$ is a distributive double p-algebra; thus, $[D] \cap C$ is open. Hence $[X)=X \cup[D)$ is open in $S(C)$. A similar argument shows that if $X$ is a clopen increasing set then $(X]$ is open. The proof is complete. 
By compactness, the chain $C$ has a minimum element $a$ and a maximum element $b$.

Lemma 7. Let $f: S(C) \rightarrow S(C)$ be the dual of a double p-homomorphism. If the minimum element of $L$ has no cover and the maximum element of $L$ is not a cover, then

$$
f\left(C \cup\left\{a_{(0)}, b_{(1)}\right\}\right)=C \cup\left\{a_{(0)}, b_{(1)}\right\} .
$$

Proof. The poset $S(C)$ is connected. Thus, for $i=0,1, f\left(C_{i}\right)=C_{i}$. Let $f(c) \in C_{0}$, for some $c \in C$. Then $f((c])=\{f(c)\}$; in particular, $f(a)=f(c)$. However, $C_{1}=\operatorname{Max}(a)$. Thus, $f\left(C_{1}\right)=\operatorname{Max}(f(a))=\operatorname{Max}(f(c))$. If $f(c) \neq a_{(0)}$ then $a_{(1)} \notin \operatorname{Max}(f(c))=f\left(C_{1}\right)$; that is, $C_{1} \nsubseteq f\left(C_{1}\right)$ which is a contradiction. Hence, $f(c)=a_{(0)}$. Similarly, if $f(c) \in C_{1}$, for some $c \in C$, then $f(c)=b_{(1)}$. We conclude that $f\left(C \cup\left\{a_{(0)}, b_{(1)}\right\}\right) \subseteq C \cup\left\{a_{(0)}, b_{(1)}\right\}$.

Choose $c \in C$ such that $c \neq a, b$. Then $c_{(1)}=f\left(d_{(1)}\right)$ for some $d \in C$. Since $d \leq d_{(1)}$, it follows that $f(d) \leq f\left(d_{(1)}\right)=c_{(1)}$. As $c \neq b$, this implies that $f(d) \leq c$. Because $c \neq a$, if $f(d)<c$ then $f(d) \in(c] \backslash\left\{c, c_{(0)}\right\}$. The element $c_{(0)}$ is minimal and less than $c_{(1)}=f\left(d_{(1)}\right)$. Hence there exists $e<d_{(1)}$ such that $f(e)=c_{(0)}$. By definition, $d_{(1)}$ covers $d$; that is, $e \leq d$. Thus $f(e)$ is an element of the decreasing set $(c] \backslash\left\{c, c_{(0)}\right\}$. This contradiction shows that $f(d)=c$. We deduce that $C \backslash\{a, b\} \subseteq f(C)$. Since the minimal element of $L$ has no cover and the maximal element of $L$ is not a cover, $a$ and $b$ are limit points of the space $\mathscr{S}(L)$. The function $f$ is continuous; thus, $\{a, b\} \subseteq f(C)$. Further, $f\left(a_{(0)}\right)=a_{(0)}$ and $f\left(b_{(1)}\right)=b_{(1)}$. In conclusion, $f\left(C \cup\left\{a_{(0)}, b_{(1)}\right\}\right)=C \cup\left\{a_{(0)}, b_{(1)}\right\}$.

LeMma 8. Let $f: S(C) \rightarrow S(C)$ be the dual of a double $p$-homomorphism. If $f \backslash C=\mathrm{id}$ then $f=$ id.

Proof. Suppose that $f\left(c_{(1)}\right) \neq c_{(1)}$ for some $c \in C$. Thus $f\left(c_{(1)}\right)=d_{(1)}$ for some $d \in C$. Since $f$ is order preserving, $c<d$; in particular, $d_{(0)} \neq c$. However, $d_{(0)}<d_{(1)}$ implies that there exists $e<c_{(1)}$ such that $f(e)=d_{(0)}$. As $e<c_{(1)}$, it follows that $e \leq c$. Hence, $d_{(0)}=$ $f(e) \leq f(c)=c$, which is a contradiction. Thus, $f\left(c_{(1)}\right)=c_{(1)}$ for $c \in C$. Similarly, $f\left(c_{(0)}\right)=c_{(0)}$ for $c \in C$. The proof is complete.

The subspace $C \cup\left\{a_{(0)}, b_{(1)}\right\}$ of $S(C)$ is a compact totally order disconnected space and is dual to the lattice $L^{+}$obtained by adding a new zero and unit to $L$. By Lemma 7, if $f: S(C) \rightarrow S(C)$ is the dual of a double p-homomorphism, then the restriction of $f$ to the subspace $C \cup\left\{a_{(0)}, b_{(1)}\right\}$ is a continuous order preserving map of this space onto itself. By H. A. Priestley [8], $f$ is dual to a $(0,1)$-lattice embedding of $L^{+}$into itself. If. $L$ is stationary (i.e. the only embedding of $L$ into itself is the identity), then the restriction of $f$ to this subspace is the identity. Consequently, by Lemma $8, f$ is the identity on $S(C)$. Thus if $L$ is stationary then the endomorphism monoid of the distributive double p-algebra corresponding to $S(C)$ has only the identity mapping (i.e. the algebra is rigid). In B. Dushnik and E. W. Miller [3], it is shown that there are $2^{2^{\mathrm{K}_{0}}}$ non-isomorphic stationary chains of carc tality $2^{\kappa_{0}}$. Under the assumption of the Generalized Continuum Hypothesis, $\Gamma$ : Rotman [11] showed that there are $\aleph_{\alpha+1}$ non-isomorphic stationary chains of cardinality $\kappa_{\alpha}$ for each successor cardinal $\kappa_{\alpha}$. A. G. Pinus [6] extended this result to the 
case when $\aleph_{\alpha}$ is singular (cf. [7]). Combining the above we obtain:

THEOREM 4. (G.C.H). If $\aleph_{\alpha}$ is an uncountable cardinal that is either a successor cardinal or a singular cardinal, then there exist $\aleph_{\alpha+1}$ non-isomorphic rigid distributive double p-algebras.

In general, finite distributive double p-algebras are better understood than infinite ones; a pattern that is repeated here. It would be of interest to know if arbitrary monoids may be represented as endomorphism monoids of distributive double p-algebras; it is felt that a positive answer to this would add to an understanding of endomorphisms of distributive lattices.

\section{REFERENCES}

1. R. Beazer, The determination congruence on double p-algebras, Algebra Universalis 6 (1976), 121-129.

2. B. A. Davey, Subdirectly irreducible distributive double p-algebras, preprint.

3. B. Dushnik and E. W. Miller, Partially ordered sets, Amer. J. Math. 63 (1941), 601-610.

4. Z. Hedrlín and J. Sichler, Any boundable binding category contains a proper class of mutually disjoint copies of itself, Algebra Universalis 1 (1971), 97-103.

5. T. Katriňák, The structure of distributive double p-algebras. Regularity and congruences, Algebra Universalis 3 (1973), 238-246.

6. A. G. Pinus, On the number of pairwise noncomparable order types, Siberian Math. J. 14 (1973), 164-167.

7. A. G. Pinus, A letter to the editor, Siberian Math. J. 17 (1977), 543.

8. H. A. Priestley, Representation of distributive lattices by means of ordered Stone spaces, Bull. London Math. Soc. 2 (1970), 186-190.

9. H. A. Priestley, Ordered topological spaces and the representation of distributive lattices, Proc. London Math. Soc. (3) 24 (1972), 507-530.

10. H. A. Priestley, The construction of spaces dual to pseudocomplemented distributive lattices, Quart. J. Math. Oxford Ser. (2) 26 (1975), 215-228. 311-328.

11. B. Rotman, On the comparison of order types, Acta Math. Acad. Sci. Hungar. 19 (1968),

12. J. Sichler, Testing categories and strong universality, Canad. J. Math. 25 (1973), 370-385.

UNIVERSITY OF MANITOBA

WINNIPEG

MANITOBA

CANADA R3T 2N2 